\title{
ASPECTOS ICÓNICOS EN LA REPRESENTACIÓN DE LOS NÚMEROS: EL CASO DE LEIBNIZ EN EXPLICATION DE L'ARITHMÉTIQUE BINAIRE (1703)
}

\author{
José Gustavo Morales \\ Universidad Nacional de Córdoba - UNC - Argentina
}

(aceito para publicação em novembro de 2016)

\begin{abstract}
Resumen
En este trabajo propongo discutir la tesis de la irreducibilidad de los aspectos icónicos en la representación en matemáticas. Más específicamente, consideraré el valor epistémico de la iconicidad para el caso de diferentes representaciones numéricas en aritmética elemental. Con este fin, presentaré un estudio de caso basado en el trabajo de Leibniz con distintos sistemas numéricos. En el caso bajo consideración Leibniz argumenta en favor de la idea de que los aspectos icónicos presentes en la notación binaria revelan relaciones estructurales de los números naturales que en otros sistemas de numeración permanecen ocultas. En consonancia con el trabajo de Grosholz (2007), concluiré que los aspectos icónicos de la representación en matemáticas son irreducibles y pueden cobrar mayor o menor relevancia dependiendo de los contextos posibles de resolución de problemas.
\end{abstract}

Palabras claves: Matemática, História, Leibniz, Representación Icónica.

\section{[THE EPISTEMIC VALUE OF ICONICITY IN ARTIHMETIC: THE CASE OF LEIBNIZ'S EXPLICATION DE L'ARITHMÉTIQUE BINAIRE (1703)]}

\begin{abstract}
In my paper I propose to discuss the epistemic value of iconicity in the case of different representations of numbers in elementary arithmetic. Recalling Peirce's distinction between iconic, symbolic and indexical signs, I will first consider the thesis of the irreducibility of iconic representation in mathematics. Here I rely on research by Grosholz (2007). I will then show that iconicity is a matter of degree depending on each context of work as well as background knowledge relevant to the problem-solving activity. To this aim, I will make use of a case study: Leibniz's uses of numerical notations in arithmetic, in particular, the preference he expresses in 1703 for the binary system.
\end{abstract}


Keywords: Mathematics, History, Leibniz, Iconic Representation.

\section{Introducción}

En este trabajo propongo discutir la tesis de la irreducibilidad de los aspectos icónicos en la representación en matemáticas. Más específicamente, consideraré el valor epistémico de la iconicidad para el caso de diferentes representaciones numéricas en aritmética elemental. Con este fin, presentaré un estudio de caso basado en el trabajo de Leibniz con distintos sistemas numéricos atendiendo a las enseñanzas que podemos derivar en el contexto de dicho estudio. A partir de ello concluiré que los aspectos icónicos de la representación en matemáticas se presentan en diferentes grados dependiendo de los contextos posibles de resolución de problemas. El motivo de la selección de mi estudio de caso es doble. En primer lugar, a lo largo de su actividad como matemático Leibniz enfatiza la importancia del razonamiento visual al tiempo que desarrolla y utiliza una variedad de herramientas que permiten desplegar aspectos icónicos fructíferos para la investigación, en particular en el dominio de la actividad de resolución de problemas. En segundo lugar, Leibniz parece discutir justamente el peculiar aspecto de la iconicidad en la representación de los números; en particular, aborda la cuestión del valor epistémico de diferentes sistemas numéricos cuando discute los beneficios del sistema binario vis-à-vis el sistema de numeración arábiga con base 10. Para Leibniz algunas notaciones son más fructíferas que otras; algunas de las virtudes que destaca el autor de los sistemas de notación es su simplicidad y su economía. En el caso bajo consideración Leibniz argumenta en favor de la idea de que los aspectos icónicos presentes en la notación binaria revelan relaciones estructurales de los números naturales que en otros sistemas de numeración, tales como el sistema de numeración arábiga con base 10, permanecen ocultas.

\section{La noción de representación de la cual parto}

En Representation and productive ambiguity in mathematic and the sciencies (2007) Emily Grosholz sostiene que las herramientas por cuyo medio el matemático lleva a cabo en análisis de los objetos de estudio son los distintos modos de representación, a saber, diagramas, fórmulas, series aritméticas, notaciones y discurso del lenguaje natural, etc.

De acuerdo con la autora las representaciones por un lado cumplen la función de designar, i. e., ellas se presentan como medios de expresión de los objetos de estudio y deben denotarlos exitosamente. Pero por el otro, la representación de un objeto no se plantea como mera reproducción de aquello que se presenta ante nosotros, sino que también constituye una herramienta para explorarlo, para examinarlo desde una perspectiva particular. Las representaciones en la investigación formal son concebidas entonces como "herramientas de trabajo" - "paper tools". Notemos aquí que la propia noción de

1 "Paper tools" es una expresión que Emily Grosholz toma de Ursula Klein (1999). 
"herramienta" ya presupone la existencia de un usuario con las habilidades requeridas para su empleo. En este punto Grosholz vincula su concepción sobre la representación con lo que define como "experiencia matemática": el análisis de los objetos de estudio que se desarrolla en el marco de una determinada tradición de investigación dentro de la cual se diseñan las herramientas requeridas para la investigación de ciertos ítems. (Cf. Grosholz 2007, 47-60) Diremos que al interior de una tradición de investigación tiene lugar una determinada "cultura de representación" en la que el matemático fue educado. Grosholz adopta del filósofo de la ciencia Jean Cavaillès la noción de "espacio combinatorio" para referir al conjunto de relaciones formales que ha sido históricamente forjado dentro de una cultura matemática desde la cual el investigador aborda los problemas que se propone resolver, lleva a cabo el análisis de sus objetos de estudio y elabora definiciones. (Cf. Cassou-Noguès, Pierre 2006)

Ahora bien, ¿cómo caracteriza Grosholz las herramientas de trabajo del matemático? En este punto la autora recupera la distinción de Peirce entre ícono, símbolo e índice. (Peirce 1885) Si bien, de acuerdo con Grosholz, un ícono representa por su semejanza con el objeto representado - como es el caso de un diagrama en la geometría de Euclides -, un símbolo representa por convención - como es el caso de una ecuación o una serie aritmética -, y un índice se presenta a los fines de establecer un orden mediante una secuencia de números o letras - como es el caso de las "fórmulas bien formadas" de Gödel -, una representación, agrega, cumple en cada caso una función que puede ser icónica, simbólica o indexical. Más aún, dado que una representación tiene lugar en un contexto determinado de la práctica del matemático, muchas veces una misma representación cumple en un caso una función preponderantemente icónica, y en otro caso la función de esa misma representación es preponderantemente simbólica. En este trabajo adoptamos la tesis de Grosholz de que toda representación aloja tanto ingredientes icónicos, simbólicos como indexicales. Para ello es preciso ante todo reconsiderar la noción de "iconicidad", la cual muchas veces suele asociarse a la noción de semejanza.

\section{La idea de representación icónica}

Examinemos ahora la idea de representación icónica. Las representaciones pueden ser icónicas, simbólicas o indexicales dependiendo de su función en los razonamientos que emplean signos en cada contexto específico de trabajo. De acuerdo con la mirada estándar decimos que las representaciones son icónicas cuando se asemejan a las cosas que ellas representan. Para el caso de la aritmética, esta caracterización resulta poco satisfactoria puesto que apela a una idea de semejanza cualitativa un tanto vaga y difícil de sostener cuando lo que está implicado es la representación de los números. Sin embargo, Grosholz argumenta que la iconicidad en matemáticas es un ingrediente irreducible:

"In many cases, the iconic representation is indispensable. This is often, though not always, because shape is irreducible; in many important cases, the canonical representation of a mathematical entity is or involves a basic geometrical figure. At the same time, representations that are 'faithful to' the things they represent may often be quite symbolic, and the 
likenesses they manifest may not be inherently visual or spatial, though the representations are, and articulate likeness by visual or spatial means". (GROSHOLZ, 2007, p. 62)

A fin de determinar si una representación es icónica o simbólica, el contexto discursivo del investigador debe ser tenido en cuenta en cada caso particular; en otras palabras, la iconicidad no puede simplemente ser leída independientemente de un contexto de uso determinado. Es posible encontrar aquí una lectura más sutil de "iconicidad" respecto a las lecturas tradicionales. Consideremos la idea de que "las representaciones articulan la semejanza por medios visuales y espaciales" en el caso de la aritmética. Grosholz destaca que incluso el razonamiento simbólico más abstracto va siempre acompañado de ciertas formas de recursos visuales. Para muchos esto puede parecer ciertamente polémico. Se adhiere generalmente a la idea de que "la forma es irreducible" en geometría para el caso de diagramas. Pero ¿cuál es el rol de la iconicidad en la representación de los números? Y más en general ¿qué debemos entender por visualización en el caso de la aritmética?

Valeria Giardino (2010) ofrece una caracterización muy útil de la actividad cognitiva de visualizar en las ciencias formales. Al visualizar, explica, estamos decodificando la información articulada que está alojada en una representación; tal articulación es un tipo especial de organización espacial que da unicidad a una representación haciéndola inteligible. En otras palabras, la organización espacial no es sólo una cuestión de desplegar algo materialmente sobre una superficie dada (un papel, una tabla, un pizarrón) sino una "espacialidad inteligible" que requiere apelar sustancialmente a conocimientos previos de base ("background knowledge"):

"(... ) to give a visualization is to give a contribution to the organization of the available information (...) in visualizing, we are referring also to background knowledge with the aim of getting to a global and synoptic representation of the problem". (GIARDINO, 2010, p. 37)

De acuerdo con esta perspectiva, la capacidad de leer o interpretar aquello a lo cual una representación refiere depende de conocimientos previos de base y de la habilidad ("expertise") del lector. Este acto cognitivo sólo puede ser exitoso si el usuario es capaz de decodificar la información encriptada en una representación pudiendo al mismo tiempo establecer una relación significativa entre la representación y el conocimiento de base relevante - que por lo general es un conocimiento implícito ("tacit knowledge"). El punto de partida de este proceso se pone en marcha con representaciones que son icónicas en un sentido rudimentario, a saber, ellas poseen aislamiento espacial y organizan la información por medios espaciales y visuales; ellas son también objetos indivisibles en la medida en que su sentido está en la unidad. Diremos a modo de síntesis, que toda representación posee "sugestividad gráfica" ("graphic suggestiveness"), una noción que Grosholz toma prestada de Nelson Goodman. 


\section{El rol de la iconicidad en la representación de los números}

A la luz de las consideraciones previas, atenderé a continuación a mi estudio de caso con el objetivo de analizar el rol que juega la iconicidad en la representación de los números naturales para el caso de la notación arábiga con base $10(0,1,2, \ldots, 9)$ y para el caso de los numerales binarios, i. e., el sistema de notación arábiga con base $2(0,1)$. En distintos fragmentos Leibniz discute ambos sistemas numéricos. (Cf. "De Progressione Dyadica": MS, 15 March 1679; C 284; GM VII, pp. 85-89) El autor los compara en relación con su utilidad para realizar cómputos y a su valor heurístico para el descubrimiento. Quisiera comenzar indagando acerca del interés que tiene la elección de una representación particular de los números en el contexto de la resolución de problemas aritméticos. En este punto me apoyo en la perspectiva de Leibniz desde el marco conceptual planteado por Grosholz (2007). De acuerdo con este marco, el uso de diferentes modos de representación en las ciencias formales nos permite explorar las condiciones de inteligibilidad, explicativas y productivas, de los objetos y problemas investigados. (Cf. Grosholz 2007, 33) Los objetos de estudio de las matemáticas son abstractos ("inteligibles"), y ellos no se presentan en forma transparente sino que son problemáticos e inagotables por lo que requieren ser analizados para clarificarlos y para desarrollar luego estructuras conceptuales ayudados por las herramientas apropiadas afines al análisis. Para el caso de la reflexión teórica acerca de los números, diversos modos de representación revelan diferentes aspectos de los objetos de estudio que pueden guiarnos al descubrimiento de nuevas propiedades y al diseño de herramientas matemáticas más elaboradas.

\section{La representación de los números}

Desde esta perspectiva consideraré ahora el caso de la representación de los números naturales. Un número natural es, escribe Grosholz, "la unidad o bien la multiplicidad de unidades en un número". (Ibid., 262) Siguiendo esta idea, una representación altamente icónica del número seis podría lucir así:

\section{IIIIII}

Por un lado, esta "imagen" exhibe la multiplicidad de unidades contenidas en el número seis. Por otro lado, la unidad del número seis está expresada por medio de una estrategia que lo diferencia del resto de los objetos que lo rodean en la página. En este caso particular, las seis barras están organizadas espacialmente para lograr este objetivo. Sin embargo, esto funciona sólo con números pequeños y si queremos representar, por ejemplo, el numero veinticuatro, la iconicidad en parte colapsa porque el lector no puede visualizar fácilmente el número que se quiere representar de esta manera. En oposición a esta representación rudimentaria de los números por medio de barras, tomemos el numeral arábigo " 6 " que no revela la multiplicidad de unidades que están contenidas en el número natural seis pero que en vez de eso exhibe la unidad del número mismo. Los numerales arábigos exhiben cada número como una unidad y sólo por esta razón esta representación es también icónica. Si lo 
que se pone en juego son grandes números, la representación por medio de barras resulta de poca utilidad y la iconicidad, si bien más débil, de los numerales arábigos se muestra más productiva en actividades de resolución de problemas tales como cómputos básicos con números. En otras palabras, la iconicidad es una cuestión de grado que depende de cada contexto de trabajo así como también del "background knowledge" relevante a la actividad de resolución de problemas. Cuando se trata de operaciones aritméticas la "sugestividad gráfica" de las barras puede no ser la última palabra mientras que la "maniobrabilidad" de los numerales arábigos, i. e., la facilidad con la que pueden ser manipulados, resulta de mayor importancia puesto que nos permite operar con precisión y facilidad. En la notación arábiga cada marca individual es "densa", en el sentido de que cada carácter codifica una gran cantidad de información.

\section{Leibniz y su preferencia por el sistema binario}

Centremos la discusión ahora en el enfoque de Leibniz sobre la notación binaria. Consideraré la exposición cuidadosa que hace de este tema en "Explication de l'arithmetique binaire", un trabajo de Leibniz publicado por la Academia Real de Ciencias de París, en 1703. Considero este caso de estudio de gran interés ya que ilumina aspectos específicos que son centrales al tópico de mi trabajo, en particular, el valor epistémico de la representación icónica de sistemas numéricos en contextos de trabajo específicos de resolución de problemas. Leibniz enfatiza lo que concibe como virtudes cognitivas de su sistema de notación favorito en aritmética, la notación binaria, la cual vuelve explícitas distintas propiedades de los números únicamente por medio de dos caracteres, a saber, 0 y 1 y las siguientes cuatro reglas: $1+1=10,1+0=1$ (suma) y 1 x $1=1,1$ x $0=0$ (multiplicación). En notación binaria cuando llegamos a 2, debemos empezar de nuevo; luego, en esta notación “dos” se escribe 10, “cuatro", 100, “ocho", 1000, y así sucesivamente.

Una y otra vez Leibniz enfatiza los valores de esta notación binaria, la economía y simplicidad del sistema por sobre todo. Toda la aritmética puede ser expresada por sólo dos caracteres y algunas pocas reglas para la manipulación de estos caracteres. Habiendo presentado las leyes de la construcción del sistema, Leibniz explica sus beneficios comparándolo con el conocido sistema decimal:

"Mais au lieu de la progression de dix en dix, j'ai employé depuis plusieurs années la progression la plus simple de toutes, qui va de deux en deux; ayant trouvé qu'elle sert à la perfection de la science des Nombres". (LEIBNIZ, 1703 - en GM VII, p. 223)

Para Leibniz, la economía y la simplicidad de su sistema binario son aspectos superadores en relación al sistema de numeración decimal arábigo. La simplicidad y facilidad con la que es posible operar con este sistema van de la mano, y mientras que en la notación arábiga siempre tenemos que recurrir a nuestra memoria, cada operación con los elementos del sistema en notación binaria se hace totalmente explícita: 
"Et toutes ces opérations sont si aisées (...) [o]n n'a point besoin non plus de rien apprendre par coeur ici, comme il faut faire dans le calcul ordinaire, où il faut sçavoir, par exemple, que 6 et 7 pris ensemble font 13; et que 5 multiplié par 3 donne 15 (...) Mais ici tout cela se trouve et se prouve de source...". (Ibid., p. 225)

Tomemos el caso de tres multiplicado por tres. A fin de resolver este caso por medio del sistema decimal, Leibniz destaca que necesitamos apelar a la memoria; debemos recordar la tabla de multiplicar por tres que nos lleva al resultado correcto, y lo mismo para cualquier numeral de 0 a 9 del sistema arábigo. Por el contrario, la misma operación hecha con el sistema binario hace siempre explícita todas las aplicaciones de las reglas para cualquier operación que realicemos. En este caso no necesitamos basarnos en la memoria sino sólo en la combinación de los caracteres los cuales se despliegan por completo en la página. Así, en la notación decimal sabemos de memoria que 3 × $3=9$ mientras que en la notación binaria la misma operación luce de esta forma:

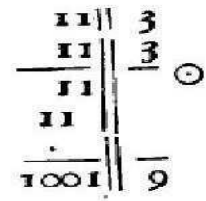

Figura 1: producto de 3 por 3 en notación binaria y arábiga respectivamente. Extraído de Leibniz, Mathematische Schriften, VII, ed. Gerhardt, 225.

donde el numeral "11" expresa el número natural tres y el numeral "1001” el número nueve.

Además, Leibniz insiste, el sistema binario revela relaciones estructurales entre los caracteres, y en el mismo escrito de 1703 señala que en virtud de la economía y la simplicidad del sistema binario podemos visualizar fácilmente las relaciones estructurales entre números descubriendo relaciones nuevas. El ejemplo que presenta aquí para ilustrar su punto es el de la progresión geométrica cuya razón es dos:

"On voit ici d'un coup d'oeil la raison d'une propriété célébre de la progression Géométrique double en Nombres entiers...". (Ibid., p. 224)

Tomemos la siguiente progresión geométrica "deux-en-deux" de números naturales: 7, 14, 28. Luego, expresamos esos números como sumas de potencia de dos. Primero, en el sistema de numeración decimal arábigo tenemos las siguientes progresiones: 
a) $4+2+1=7$

b) $8+4+2=14$

c) $16+8+4=28$.

En segundo lugar, procedemos a descomponer $a, b$ y $c$ en potencias de dos:

a') $2^{0}+2^{1}+2^{2}=2^{0} \cdot 7$

b') $2^{1}+2^{2}+2^{3}=2^{1} \cdot 7$

c') $2^{2}+2^{3}+2^{4}=2^{2} \cdot 7$

En el primer caso, ninguna de las tres líneas nos da información sobre el patrón que conduce a $a, b, c$. Más bien, las tres líneas requieren familiaridad con todos los elementos del sistema así como también con la operación en cuestión (suma).

De manera similar, en el segundo caso, las tres líneas no dan ninguna información acerca del patrón que subyace a la progresión. En cada una de las tres líneas, el lado derecho de $a^{\prime}, b^{\prime}, c^{\prime}$ no indica el resultado. Debemos encontrar cómo expresar el resultado como potencias de dos. En los tres casos vemos que el resultado no puede ser expresado como una potencia de dos y se hace necesario introducir un nuevo elemento: el factor 7 . Por supuesto, también debemos conocer la tabla de multiplicar por siete.

Volvamos ahora a la notación binaria y consideremos cómo la distribución espacial de $a, b$ y $c$ es expresada en el sistema binario:

$\begin{array}{rrr}100 & 1000 & 10000 \\ 10 & 100 & 1000 \\ 1 & 10 & 100 \\ - & & \\ & & 11100\end{array}$

Figura 2. Progresión geométrica de razón dos expresada en notación binaria.

En oposición al sistema decimal de numerales arábigos, dentro del sistema binario no es necesario analizar el caso en dos pasos separados. Esto es así debido a que los caracteres y el orden que ellos exhiben sobre la página hacen visibles los patrones que subyacen a la progresión. Sólo necesitamos conocer cómo funcionan las reglas para la adición y el sistema de caracteres (0 y 1$)$.

Finalmente, Leibniz señala otra de las características de los binarios, esta vez en relación con la construcción del sistema. Es la simplicidad y economía del binario que, según él, exhibe una periodicidad y un orden notable. Para acentuar este punto, el autor hace hincapié una vez más en los aspectos visuales y en la configuración espacial de los caracteres: 
"(...) les nombres étant réduits aux plus simples principes, comme $0 \& 1$, il paroît partout un ordre merveilleux. Par exemple, dans la Table même des Nombres, on voit en chaque colonne régner des périodes qui recommencent toujours". (Ibid., 226)

Leibniz coloca en un mismo grupo los números que caen bajo $2^{1}, 2^{2}, 2^{3}$, etc. Incluyo aquí un segmento de una tabla más grande utilizada por Leibniz para mostrar tres grupos de números (delimitados por líneas horizontales y verticales), a saber, 01; 2, 3 y 4-7. Cada grupo es un ciclo que se itera en el siguiente ciclo, y así ad infinitum como podemos ver fácilmente (Figura 12). ${ }^{2}$

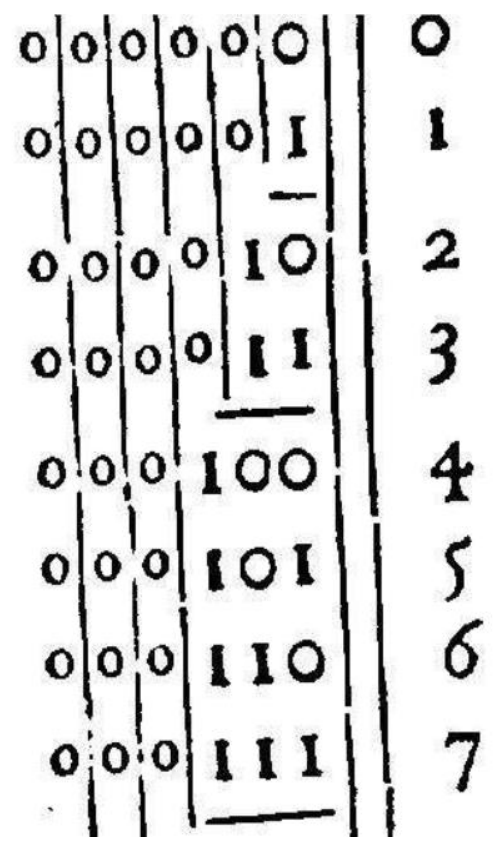

Figura 3. Leibniz, Mathematische Schriften, VII, ed. Gerhardt, p. 225.

\section{Comentarios finales}

Para Leibniz la investigación matemática comienza con la búsqueda de signos adecuados ("caracteres") y el diseño de buenas notaciones (o "características") por medio de los cuales las relaciones estructurales de los objetos de estudio puedan ser exploradas; una buena

${ }^{2}$ Leibniz, GM VII, 224.

RBHM, Vol. 16, no 32, p. 41-51, 2016 
"característica" debería permitirnos descubrir diferentes aspectos de las cosas por medio de un tipo de razonamiento con signos. Cuando Leibniz destaca esto en un breve texto de 1683-1684, su ejemplo de una "característica más perfecta" es la notación binaria vis-à-vis el sistema decimal. (Cf. C 284) Para entender la preferencia de Leibniz por el sistema binario, recordamos aquí, es útil centrarse en la importancia del razonamiento visual en contextos de resolución de problemas. Según Leibniz, las actividades de resolución de problemas y el descubrimiento de nuevas propiedades son las metas del análisis matemático en el caso de la teoría de números, una de las áreas de investigación a la que dedicó una gran cantidad de tiempo y esfuerzo. Tales objetivos motivan fuertemente el diseño de sistemas de notación con el fin de obtener resultados fructíferos. Como ya se ha señalado, para Leibniz el sistema binario se caracteriza por su sencillez y economía de modo que en cada operación cada elemento ("0", "1") es visible para el ojo, sin necesidad de confiar en la memoria como sucede en el caso de las operaciones con números arábigos. Leibniz observó que en ciertos contextos de trabajo la distribución espacial de estos elementos binarios revela patrones que son relevantes para la resolución del problema que está siendo tratado, $\mathrm{o}$ al descubrimiento de nuevas propiedades que de otro modo permanecerían ocultas. Tal es el caso de la progresión geométrica "deux-en-deux", la cual, como acabamos de presentar, se puede visualizar fácilmente sólo cuando se expresa por medio del sistema binario. No hay duda de que, para propósitos prácticos de la vida cotidiana el sistema decimal de números arábigos resulta más útil para el cálculo, sin embargo, Leibniz estaba fascinado con el sistema binario como facilitador de estructuras algorítmicas de la misma manera que lo estaba con las estructuras numéricas que permitían el cálculo de valores finitos mediante series infinitas de números racionales.

Para concluir, una de las cosas que podemos aprender del presente estudio de caso es que en el cálculo numérico siempre necesitamos sistemas de signos o caracteres, pero algunos de ellos son más fructíferos que otros, algunos facilitan el cálculo más que otros dependiendo en cada caso del contexto de trabajo; pero más allá de las virtudes epistémicas específicas que puedan tener, todos ellos incluirán importantes aspectos icónicos relevantes para la cognición. Esta conclusión, en particular, pone en duda la vieja idea de que el trabajo con estructuras algorítmicas - computación con números - es un asunto puramente mecánico que excluye la iconicidad.

\section{Bibliografia}

BREGER, Herbert. 2008. The art of mathematical rationality. En Leibniz: what kind of rationalist? M. Dascal (ed.), Springer Science+Business., pp. 141-154.

BREGER, Herbert. 1992. Tacit knowledge in Mathematical Theory. En The space of mathematics - Philosophical, Epistemological, and Historical Explorations, Echeverría, Andoni y Mormann (eds.), Walter de Gruyter, Berlin - New York.

CAMPOS, Daniel. 2009. Imagination, Concentration and Generalization: Peirce on the Reasoning Abilities of the Mathematician. Transactions of the Charles S. Peirce Society: A Quarterly Journal in American Philosophy, volúmen 45, número 2, 135-156.

CASSOU-NOGUÈS, Pierre. 2006. Signs, figures and time: Cavaillès on 'intuition' in mathematics. Theoria 55: 89-104. 
GIARDINO, Valeria. 2010. Intuition and Visualization in Mathematical Problem Solving, Dordrecht, Springer, Topoi (2010) 29:29-39.

GROSHOLZ, Emily. 2007. Representation and productive ambiguity in mathematics and the sciences. Oxford New York.

LEIBNIZ, G. W.: Selección de fragmentos, obras \& correspondencia (Ed. Akademie Verlag, Berlin) y Gerhard Edition: Philosophische Schriften I-VII \& Gerhard: Mathematische Schriften I-VII), Olms (reimpresión) 1961.

PEIRCE, C. S. 1885. On the Algebra of Logic: A Contribution to the Philosophy of Notation. The American Journal of Mathematics, 7 (2) (1885), 180-202; repr. en Collected Papers of Charles Sanders Peirce, eds. C. Hartshorne and P. Weiss (Cambridge: Harvard University Press, 1931-), Vol. 3, pars. 359-403.

José Gustavo Morales

Universidad Nacional de Córdoba / Consejo

Nacional de Ciencia y Técnica - UNC / CONICET

- Argentina

E-mail: gust.914@gmail.com 